\section{Cahiers de Narratologie}

Analyse et théorie narratives

8 | 1997

Création de l'espace et narration littéraire

\title{
Le « Haut et le bas » $:$ repères spatiaux dans $L a$ montagne magique et Gel
}

\section{Marc Moser}

\section{(2) OpenEdition}

1 Journals

\section{Édition électronique}

URL : http://journals.openedition.org/narratologie/11608

DOI : 10.4000/narratologie. 11608

ISSN : 1765-307X

Éditeur

LIRCES

\section{Édition imprimée}

Date de publication : 1 décembre 1997

Pagination : 277-284

ISBN : 291089746X

ISSN : $0993-8516$

\section{Référence électronique}

Marc Moser, "Le « Haut et le bas » : repères spatiaux dans La montagne magique et Gel », Cahiers de Narratologie [En ligne], 8 | 1997, mis en ligne le 15 décembre 2020, consulté le 25 février 2021. URL:

http://journals.openedition.org/narratologie/11608; DOI : https://doi.org/10.4000/narratologie.11608

Ce document a été généré automatiquement le 25 février 2021.

Article L.111-1 du Code de la propriété intellectuelle. 


\title{
Le « Haut et le bas » : repères spatiaux dans La montagne magique et Gel
}

\author{
Marc Moser
}

1 Notre contribution sera d'ordre comparatif. Nous mettrons en parallèle La Montagne Magique, œuvre majeure de Thomas Mann et Gel-Frost de Thomas Bernhard. Les deux écrits datent respectivement de 1924 pour ce qui concerne le livre de Thomas Mann et de 1963 quant au premier grand roman qu'est Gel de Thomas Bernhard.

2 «Le haut et le bas » revêtent dans les deux romans des valeurs spatiales, ontologiques et symboliques. Nous tenterons d'en dégager les articulations, les affinités et les différences. Les deux dates de parution se situent à 39 ans d'intervalle. La période de gestation de La Montagne magique s'étend sur douze ans 1912-1924 et débouche sur la grande guerre de 1914, coup de tonnerre qui rappelle à la dure réalité conflictuelle des hommes. Descente rapide des cimes enneigées suisses et réveil brutal de la rêverie des hauteurs. C'en est fini du songe et des discussions intellectuelles et idéologiques.

Un des éléments récurrents de Gel constitue la seconde guerre mondiale et ses terribles conséquences, véritables perturbations du psychisme humain. Le haut et le bas y paraissent en une constante interaction. Ces deux contraires coöncident dans une identité qui rappelle la célèbre formule d'Héraclite :

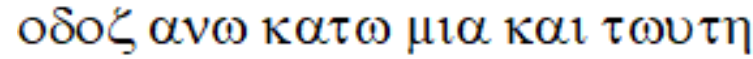

5 La localité de Weng où se déroule le roman est «située bien haut, mais pourtant toujours comme dans les profondeurs d'une gorge» (p. 10).

6 Nous verrons alors que l'opposition entre le haut et le bas s'efface progressivement pour aboutir à un pessimisme grandissant sans rêve ni espoir, sous le seul signe de la 
nécessité de vivre cette existence haïe où coïncident les contraires, attachement et aversion, tout comme le haut et le bas.

Thomas MANn, La Montagne magique, Berlin 1924, 1912-1924.

8 Au cœur de cette œuvre couronnée par le Prix Nobel en 1929 loge l'intérêt pour la maladie, le pathologique, la déchéance, la mort. Celui-là même exprime l'attachement à la vie. Cette visée rejoint ce que Nicolas de Cusa (1401-1464) qualifia dans son écrit majeur De docta ignorantia de «coïnci-dentia oppositorum», autrement dit les contraires se complètent et demeurent inséparables.

Les deux protagonistes du roman de Thomas Mann s'appellent :

1. Hans Castarp fils fortuné issu d'une famille patricienne de Hambourg, il vient du bas, de la plaine.

2. Joachim Ziem ?en, cousin du précédent, poitrinaire, se soigne dans un sanatorium de Davos, vit sur les hauteurs alpines d'où il domine les cimes enneigées.

10 Le premier rend visite au second et ce faisant, après avoir obtenu son diplôme d'ingénieur, il succombe au charme et à la magie de la montagne suisse qui le retient sept ans dans ses rets. Il s'agit d'un ensorcellement hermétique du monde du bas qui paraît provisoirement évacué. Il s'agit de la crise d'une maladie qui débouche ou bien sur la vie ou bien sur la mort.

11 Hans Castorp, le visiteur venu de l'estuaire de Hambourg, quasi niveau de la mer du Nord, tombe à son tour effectivement malade. De surcroît il est pris par le vertige de l'ambiance du sanatorium de luxe où le macabre et la fièvre d'illuminé font tourner la tête.

12 Nous avons à faire à un roman à la fois d'initiation et de formation - Bildungsroman émaillé d'éléments d'aventure et picaresques

13 Le titre même du roman

14 La Montagne magique - Der Zauberberg - s'inspire de Goethe: Faust la nuit de «Walpurgis». Le nombre sept représente une récurrence voulue dans l'œuvre: la durée du séjour à Davos est de sept ans (de 1907 à 1914). Sept chapitres comptent et content l'histoire de Hans Castorp. Dans cette auberge alpine, ce dernier mange successivement à sept tables. Son mentor majeur s'appelle Settembrini.

15 L'éloignement spatial de la réalité foisonnante et trépidante qu'est celle d'un port comme Hambourg produit progressivement la perte de la notion du temps. L'inactivité prolongée sur la montagne magique, la stupidité quotidienne de la position horizontale des patients, cette existence monotone, - étymologiquement tendue seulement vers la médication - caractérisent l'inanité d'une vie en altitude oisive et oiseuse.

16 Nous observons alors le schéma en chiasme suivant: la position verticale du bien portant actif de la plaine s'oppose à la position horizontale du malade transporté en altitude où il végète en attendant de guérir ou de mourir.

17 Hans Castorp assistera en effet à la mort difficile et douloureuse de Joachim Ziem ?en, son cousin, rêvant de son vivant d'une brillante carrière d'officier qu'il fut de formation. Est-ce là une allusion à peine perceptible à la disparition du militarisme au moins prussien? La question mérite d'être posée et demeure ouverte. En tout cas l'officier ne meurt pas debout comme au combat guerrier.

18 Il sera témoin d'un autre décès, celui lors d'un duel de Naphta, patronyme suggestif; le naphte, pétrole, huile noire de la terre, d'un agitateur dangereux, fanatique ascétique, 
eschatologue terroriste prônant un marxisme radical et sans concession. Naphta annihilera lui-même son existence absurde.

En effet Settembrini défenseur convaincu d'un humanisme éclairé, progressiste et optimiste se dispute avec Naphta au point d'en arriver au duel au cours duquel Naphta se tire lui-même une balle dans la tête. Cette issue létale revient-elle à une condamnation sans appel des agissements et menées du terroriste fanatique? Sans aucun doute. On peut y voir également la prédiction du sort réservé tôt ou tard au léninisme-marxisme totalitaire que Thomas Mann semble redouter. L'affrontement violent et fatal entre les deux protagonistes aboutit donc à la survie de l'humaniste Settembrini qui revêt de par sa stature la mission de propager ses idées-phares de valeurs humaines. C'est que l'humain est pour Thomas Mann une conquête personnelle. Celle-ci résulte d'une lutte contre les tentations de l'irrationnel, de l'instinctif ; c'est par là qu'il se situe dans la lignée du classicisme. Écrire revient, pour Thomas Mann, à jouer avec les simulations afin de dominer le réel, de se guérir des blessures qui en proviennent.

Comment ne pas penser à la fin de La Montagne magique de Thomas Mann, où Hans Castarp retourne à la plaine, à la réalité du bas après avoir échappé de justesse à l'envoûtement périlleux des montagnes?

21 Qu'en conclure? Les hauteurs fascinantes incitant à des envolées philosophiques, des discussions idéologiques et théoriques sont-elles à jamais irrémédiablement coupées de la réalité du bas, du terre à terre où règnent la dure action le concret prosaïque ?

Par rapport à La Montagne magique de Thomas Mann, le récit Gel-Frost de Bernhard semble sinon abolir, du moins fortement atténuer l'antinomie bas/haut qui se confondent en quelque sorte. Les deux mondes quasiment indifférenciés servent à confirmer l'absurde dont le règne s'avère indépendant des dénivellations.

23 La vision de Thomas Bernhard paraît rejoindre celle déjà exprimée par Héraclite, à savoir le chemin vers le haut et le bas est un et le même.

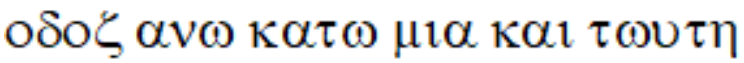

D'emblée Bernhard fait dire au peintre Strauch que pour celui arrivé en haut il s'avère que le haut n'existe pas (Gel, p. 20). Plus loin, Strauch affirme même que le haut devient le bas; il en résulte que les deux niveaux s'identifient et sont interchangeables, se valent.

1963 Frost-Gel de Thomas Bernhard

Ce premier grand roman valut à Thomas Bernhard en 1963 la percée littéraire. Y apparaît déjà la structure ternaire fondamentale et caractéristique de l'œuvre toute entière.

La durée s'étend sur $3 \times 3 \times 3=27$ journées. Le nombre 27 constitue pour le moins un hommage discret aux 27 liasses de la copie des Pensées de Pascal. Ce livre philosophique occupe une place prééminente. En effet un des trois personnages clef, le peintre Strauch ne se sépare jamais de ce recueil.

28 La première triade de ce roman comprend deux frères ennemis, les Strauch, l'un est en fonction comme médecin assistant à l'hôpital en bas à Schwarzach, l'autre se consacre à 
la peinture à Weng; cette petite localité est située tout en haut du Val de Salzach et représente le monde, la sphère du haut. Le médecin du fond de la vallée envoie un étudiant, interne de l'hôpital de Schwarzach chez son frère avec lequel il est brouillé pour le faire observer. L'interne remplit les fonctions de narrateur anonyme et simultanément de tiers intermédiaire chargé d'établir un lien entre les deux frères fâchés.

29 À côté des études médicales l'interne est amené à s'occuper d'états de fait et de possibilités situés en dehors du corps humain - extra-charnel - au ?erfleischlich - à explorer quelque chose d'inexplorable, d'impénétrable - etwas Unerforschliches zu erforschen - . Ce domaine situé hors la chair, dépourvu de cellules pourrait parfaitement incarner tout ce qu'il existe et non pas inversement.

Ces considérations et réflexions sont propres au monde du haut qui stimule l'activité cérébrale élevée localisée précisément sur les hauteurs.

31 Y fait face et s'y oppose le monde du bas à l'activité ralentie, diminuée voire parfois paralysée du cerveau.

La deuxième triade peuple ce monde bas qui se déroule et défile dans une auberge : s'y côtoient la femme-aubergiste décrite par l'interne comme véritable Pandore, mal féminin ; elle est séparée de son mari détenu de par la faute d'elle-même. Le troisième élément en constitue l'équarrisseur et également fossoyeur du village Weng. $\mathrm{Ce}$ dernier, en l'absence du maître de céans, fait figure d'amant de la femme esseulée.

L'époque se situe dans l'après-guerre de l'Autriche sans doute encore occupée, donc avant 1955. Les souvenirs des atrocités et souffrances de guerre sont encore vivaces; cette région montagneuse des environs de Weng constituait le refuge des bombardements de Salzbourg. Le peintre Strauch et sa sœur s'y étaient mis en sécurité. Et lui d'affirmer en justifiant le titre du roman - Gel-Frost - que « tout périt à cause du froid - et de la froideur - même les visions oniriques » (p. 27).

Les dialogues entre l'interne, le narrateur, et le peintre occupent la majeure partie du roman. Ces deux protagonistes sont caractérisés et déterminés de par leur lecture exclusive, à savoir, le peintre ne porte sur lui que "son Pascal» (p. 21) quant à l'étudiant en médecine, l'observateur de liaison entre les deux frères Strauch, il ne lit qu'un livre de Henry James (1843-1916). L'œuvre du romancier anglo-américain fait apparaître le leitmotiv et la trame, dans une optique tchéchovienne, de l'angoisse de passer à côté de la vie et de la quête du destin individuel et national. Le caractère humain, dans les écrits de James semble déterminé par l'événement et l'action principalement.

35 L'objectif déclaré du peintre Strauch est, expressis verbis, de construire entre DEUX CARACTÈRES un TROISIÈME et ainsi de suite (p. 21). Dans la vision pascalienne, Pensée 1670, Strauch comprend l'homme comme un être ambivalent arraché à l'unité et à la simplicité divines, moitié corps, moitié esprit, corrompu qu'il est, la quête de l'unité de la nature l'anime. Ce personnage égocentrique et entièrement replié sur lui-même constitue la réplique du grand-père bien-aimé, substitut du père de Thomas Bernhard.

L'aïeul de l'écrivain fut un monstre d'égoïsme ne vivant que pour ses écrits voués quasiment à l'échec. Quel défi pour le petit-fils que de réparer l'insuccès douloureux par une œuvre lyrico-romano-théâtrale célèbre et traduite dans une vingtaine de langues. 
37 L'interne ou le carabin, après avoir rédigé et envoyé six rapports d'observation sur le peintre Strauch au frère, le médecin, le $27^{\mathrm{e}}$ jour redescend à Schwarzach - toponyme parlant funeste signifiant "eau noire, sombre", situé au fond de la vallée. Dans le journal local, le Demokratische Volksblatt où le jeune Thomas Bernhard était chroniqueur judiciaire, le carabin découvre la disparition dans la neige de G. Strauch, sans profession et demeurant à Weng. confondent, s'avèrent interchangeables le monde en altitude perd ses illusions, est présenté aussi décevant et absurde que celui du plat pays. Après la deuxième guerre mondiale les effets dévastateurs de la pire des folies des hommes demeurent omniprésents, indélébiles. publication respectives. Après l'holocauste ou la shoah la vision existentielle et ontologique changea fatalement et Thomas Bernhard s'en fait l'artisan et l'écho.

\section{BIBLIOGRAPHIE}

BERNHARD Thomas : Frost, Insel Verlag Frankfurt am Main, 1963, suhrkamp taschenbuch Heraklit : Fragmente, Ernst Heimeran Verlag, München, 1965.

MANN Thomas : Der Zauberberg, G.B. Fischer, Frankfurt am Main, 1963.

PASCAL Blaise : Les Pensées, Cuuvres Complètes, Gallimard, La Pléiade n 34, Paris, 1960. 
AUTEUR

MARC MOSER

Université de Nice-Sophia Antipolis 